\title{
Methodology for Real-Time Evaluation of Geographic Health Care Resource Allocation: Iwate Prefectural Hospitals
}

\author{
Xinhe Li", Kazunori Kawamura \\ Graduate School of Information Sciences, Tohoku University, Sendai, Japan
}

Email address:

li.xinhe.q2@dc.tohoku.ac.jp (Xinhe Li)

${ }^{*}$ Corresponding author

\section{To cite this article:}

Xinhe Li, Kazunori Kawamura. Methodology for Real-Time Evaluation of Geographic Health Care Resource Allocation: Iwate Prefectural Hospitals. Science Journal of Business and Management. Vol. 9, No. 2, 2021, pp. 55-61. doi: 10.11648/j.sjbm.20210902.11

Received: April 20, 2021; Accepted: May 14, 2021; Published: May 24, 2021

\begin{abstract}
The effective utilization and assessment of medical resources have become a common concern for scholars in various countries due to the impact of the COVID-19 pandemic. This study deals with a method to monitor medical resource allocation in real-time and verify the effectiveness of the proposed method with actual data. In this work, we selected Iwate Prefecture in northeastern Japan based on the geographical characteristics and social environment. By collecting data from the Japanese Ministry of Land, Infrastructure, Transport, and Tourism and Welfare (MLIT), we clustered population centers in Iwate Prefecture, and found the clustering centers in densely populated areas from the k-means algorithm. Subsequently, to compare the distribution of county-level medical resources across different secondary care areas, we selected the indicators of Iwate Prefectural Hospitals from the Hospital Intelligence Agency. We classified 19 prefectural hospitals in Iwate Prefecture into four different clusters using the spectral clustering algorithm. The clustering results revealed that all hospitals close to the " clustering centers in densely populated areas" were Iwate prefectural disaster stronghold hospitals. Moreover, we found that these hospitals performed well in operational indicators. Only the Ninohe prefectural hospital in the Ninohe medical area was found not located in a population center. However, it still performs well in terms of business indicators since the Ninohe medical area has a high proportion of public hospitals and the Ninohe prefectural hospital plays an important role. Hence, the government should fully consider geographical characteristics when considering hospital restructuring. We used a real data set to demonstrate the validity of the proposed technique, providing a theoretical basis for the government's healthcare policy.
\end{abstract}

Keywords: Medical Resources, Real-time Evaluation Method, K-means Algorithm, Spectral Clustering Algorithm, Iwate Prefectural Hospitals

\section{Introduction}

The Japanese healthcare system is characterized by a large surplus of hospital beds $[1,2]$. Presently, Japan still has the highest number of hospital beds in the world (13 beds per 1,000 inhabitants, compared to 2.9 beds in the United States and 2.5 beds in the United Kingdom) [3]. Considering the overall low bed occupancy and a declining population in Japan, the business conditions of public hospitals in many areas are deteriorating owing to declining hospital revenues. While the demand for medical care is increasing, it becomes increasingly difficult to maintain the financial resources for medical care, local governments, hospital administrators.
Hence, it is essential to consider how to ensure the survival of the hospitals. Recently, there has been an increasing awareness of the importance of rational allocation of medical resources taking into account the changes in the demand for medical services of the regional population, which is a scientific decision [4]. Developed countries are also affected by changes in demand owing to aging populations and increasing life expectancy [5, 6]. Since COVID-19 causes significant economic, medical, and even social disruptions around the world, the effective use of medical resources has attracted experts and scholars' attention worldwide [7, 8].

From a macro perspective, the consolidation of health care resources creates the same problem of uneven distribution of 
health care resources, while having a positive effect on the overall economy. This is almost irreversible owing to the high investment involved [9]. For example, in 1999, the merger of municipalities in the Heisei era in Japan affected the location of new hospitals, which in turn led to the installation of multiple hospitals in a given municipality. It may also decrease the number of hospitals in a considered area, which often resulted in dissatisfaction among residents [10]. As a result, there is disagreement about hospital mergers owing to administrative causes. To date, despite the importance of the rationality of hospital mergers, there is still little evidence on the rationality of hospital mergers to present reasonable and robust evidence to verify the rationality of health care mergers for resident care. Most previous studies suffer from a lack of standardized measures and significant design limitations. They failed to find a reasonable and objective method to address this issue.

Since the spatial configuration and accessibility of medical facilities are of great practical and theoretical significance in assessing the current status of medical resources [11], we first used a facility configuration model to assess the locations of county hospitals. In selecting the facility configuration model, we assumed that all facility users choose the nearest county hospital and consider the principle of "fairness" for minimizing the sum of the distance of users utilizing the same facility. The center of each user set is the arrangement of facilities obtained by this method. Thus, we used the k-means algorithm to solve this problem because of its low time complexity and suitability for large-scale data [12]. Second, to compare the size of prefectural hospitals in the different secondary medical areas, we selected several operational indicators of hospitals based on the Japan Hospital Information Bureau reflecting the overall performance of hospitals [13]. For model selection, we chose the spectral clustering model, with the characteristics of adaptability to data distribution and good performance in dealing with similar data [14]. Hospital operating indicators were used as the input variables of the model. In our work, we utilized the medical resources of Iwate Prefecture for the study. Iwate Prefecture is the second largest prefecture in Japan and has many mountains. The transportation environment between cities is not good and it is not convenient for hospitals to visit neighboring areas. Regarding the number of public hospitals, Iwate prefecture has a high proportion of public hospitals among the 47 prefectures in Japan. It has the most significant number of prefectural hospitals and has historically developed a unique prefectural hospital network [15]. Hence, the importance of prefectural hospitals is inferred.

There were two primary purposes in the present work. First, we proposed a method to assess the healthcare resources in a particular region considering the changing environment. The primary evaluation index must be used as a dynamic variable so that the healthcare system can be assessed in real-time during later assessments, even in case of emergencies. The second purpose is to investigate the resource allocation of hospitals in Iwate Prefecture, Japan.

\section{Data Sources}

\subsection{Spatial Data}

We chose the 2015 Iwate population cluster boundary data as the primary spatial data downloaded from the Ministry of Land, Infrastructure, Transport, and Tourism website. Then, we converted these data into shapefiles by reading the spatial data in GeoJSON format. At the same time, we obtained the coordinate points of the boundaries of various clusters (Figure 1).

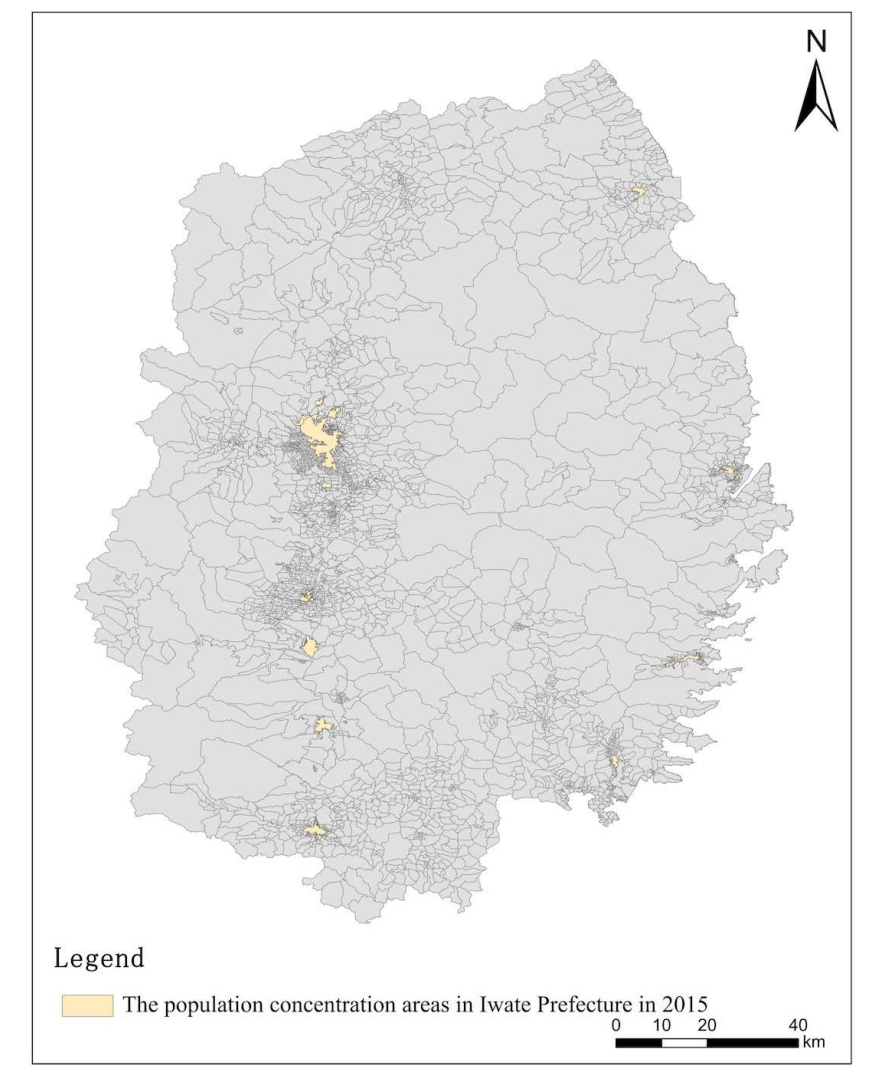

Figure 1. The population concentration areas in Iwate Prefecture in 2015.

Source: Ministry of Land, Infrastructure, Transport and Tourism (MLIT) https://www.mlit.go.jp/

\subsection{Hospital Indicator Data}

To compare the distribution of medical resources among prefectural hospitals in various areas of Iwate Prefecture, we clustered the prefectural hospitals in Iwate Prefecture. The clustered hospitals had more remarkable similarities within the same class and less similarity among different classes. We compared the distribution of medical resources at the prefectural level in different secondary medical areas in this regard.

The basic information about the hospital was based on the website of the Hospital Intelligence Agency. Considering the availability of data and preventing multicollinearity, we collected four indicators reflecting different hospital aspects (Table 1). 
Table 1. The indicators of prefectural hospitals in Iwate Prefecture.

\begin{tabular}{lllll}
\hline & Number of beds & $\begin{array}{l}\text { Number of patients } \\
\text { admitted to the hospital }\end{array}$ & Number of physicians & Number of nurse practitioners \\
\hline Otsuchi & 50 & 25 & 5 & 34.4 \\
Kamaishi & 272 & 187 & 24 & 187.6 \\
Ninohe & 263 & 169 & 49 & 178.2 \\
Kuji & 210 & 4240 & 5.5 & 260.8 \\
Ofunato & 489 & 30 & 58.8 & 33.4 \\
Takata & 60 & 59 & 7 & 42 \\
Towa & 68 & 272 & 7.5 & 277.9 \\
Isawa & 346 & 247 & 66 & 232.4 \\
Iwai & 315 & 350 & 75.4 & 587.8 \\
Central & 685 & 30 & 182.4 & 333 \\
Chubu & 434 & 71 & 100 & 146.8 \\
Ichinohe & 199 & 114 & 12 & 83.7 \\
Esashi & 122 & 85 & 13.3 & 120.5 \\
Senmaya & 152 & 223 & 9 & 117.6 \\
Tono & 122 & 22 & 9.9 & 192 \\
Miyako & 334 & 16 & 43 & 28.7 \\
Yamada & 50 & 43 & 3 & 29 \\
Daito & 40 & 59 & 5.3 & 46.5 \\
Karumai & 99.4 & 8.4 \\
\hline
\end{tabular}

(Part-time physicians converted to full-time physicians)

Source: Hospital Intelligence Agency https://hospia.jp/

\section{Methods}

\subsection{Facility Configuration Model and K-means Algorithm}

\subsubsection{Facility Configuration Model}

The location assignment model is a method to determine the location of facilities, by which spatially distributed demand sets can be assigned to facilities [16, 17, 18]. In previous studies, numerous facility allocation models were proposed. There are various ways to classify these models. Generally, facility allocation models are classified into continuous and discrete models [19]. In this paper, we used a continuum-type facility configuration model to explore the optimal location of hospitals. It is assumed that the facilities can be chosen at any location on a continuous plane [20]. The objective function is as follows:

$$
\sum_{\mathrm{i}=1}^{\mathrm{N}} \min _{1 \leq \mathrm{m} \leq \mathrm{k}}\left\{\left\|\mathrm{h}_{\mathrm{m}}-\mathrm{x}_{\mathrm{i}}\right\|^{2}\right\}
$$

where $\mathrm{N}$ represents the total population of Iwate Prefecture, $\mathrm{m}$ refers to the $\mathrm{m}$-th hospital, $\mathrm{k}$ is the total number of prefectural hospitals, $i$ refers to the $i$-th user, $x_{i}$ is the latitude and longitude coordinates of the user, and $h_{m}$ is the latitude and longitude coordinates of the prefectural hospital. According to O'Kelly (1992), a clustering algorithm could be utilized for this model [21].

\subsubsection{K-means Algorithm}

The k-means algorithm is a non-hierarchical clustering algorithm proposed by MacQueen et al. (1967). It is one of the most popular methods in unsupervised machine learning [22]. It is the algorithm that can divide $\mathrm{N}$ samples into $\mathrm{K}$ clusters. The algorithm minimizes the sum of squares within a cluster.

The k-means algorithm was applied in various fields (1970)
[23, 24] because it is simple, easy-to-understand, and practical. However, its drawbacks are also more apparent. For example, the value of $\mathrm{k}$ must be determined in advance. Considering this drawback, we conducted in-depth studies for different problems. For example, the optimal solution is chosen by multiple iterations [25]. This method has limitations to deal with numerous data, however, it can obtain clustering results close to reality. In this paper, the value of $\mathrm{k}$ was set based on the number of prefectural hospitals in Iwate Prefecture (when the number of hospitals is not references, $\mathrm{k}$ value is set to 19). To obtain more scientifically accurate clustering results, we decided to include the confirmation of data visualization and consider the evaluation index of the clustering results.

In this paper, the effectiveness of clustering was evaluated based on the Calinski-Harabasz value in equation (2):

$$
\mathrm{CH}(\mathrm{k})=\frac{\operatorname{trB}(\mathrm{k}) /(\mathrm{k}-1)}{\operatorname{trW}(\mathrm{k}) /(\mathrm{n}-\mathrm{k})}
$$

B $(\mathrm{k})$ is the variance between clusters, $\mathrm{W}(\mathrm{k})$ represents the variance within clusters, $\mathrm{N}$ is the number of data, and $\mathrm{k}$ shows the number of clusters. The larger the Calinski-Harabasz value, the better the clustering effect [26]. In this paper, the number of iterations of the k-means algorithm is set to 5000. The maximum Calinski-Harabasz value is chosen to avoid falling into a local optimum solution.

\subsection{Hospital Business Index Clustering Based on Spectral Clustering Algorithm}

Following the identification of clustering centers in population concentrations, we clustered the prefectural hospitals in Iwate Prefecture. The spectral clustering is the second method we applied. The spectral clustering algorithm 
is a new research hotspot in machine learning in recent years. It is based on the theory of spectral graphs. Its essence is to transform the clustering problem into the problem of optimal division of graphs.

Since spectral clustering includes the connection relation of graphs, it can realize the partitioning of graphs well-compared with traditional clustering algorithms, such as k-means. Spectral clustering is more appropriate for clustering data in multiple spaces. In this study, the spectral clustering algorithm is proper since the selection of hospital metrics should reflect different aspects of the hospital. The process is represented as follows: First, before clustering, we normalized the data via Equation (3):

$$
\mathrm{Z}^{*}=\frac{\mathrm{Z}-\mathrm{Z}_{\min }}{\mathrm{Z}_{\max }-\mathrm{Z}_{\min }}
$$

$\mathrm{Z}^{*}$ is the normalized value and $\mathrm{Z}$ represents the original data value; $Z_{\min }$ is the minimum value in the dataset, and $\mathrm{Z}_{\max }$ shows the maximum value in the dataset.

We utilized the normalized spectral clustering algorithm to cluster the data points [14]:

Table 2. Normalized spectral clustering.

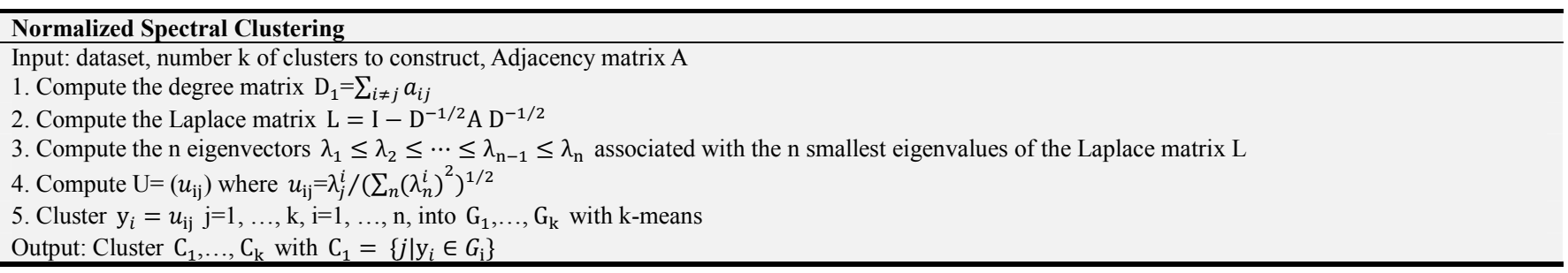

\section{Results}

\subsection{Clustering Results of the Spatial Data}

The clustering results of the population by the k-means algorithm are presented in Table 3 . By repeatedly setting the initial values, good clustering results were obtained. In this work, the center of clustering represents the appropriate location of the county hospital. Comparing the calculated results with the actual hospital locations, it is observed that Prefectural Central Hospital, Prefectural Chubu Hospital, Prefectural Isawa Hospital, Prefectural Iwai Hospital, Prefectural Kuji Hospital, Prefectural Miyako Hospital, Prefectural Kamaishi Hospital, and Prefectural Ofunato Hospital are closer to the cluster centers (Figure 2).

Table 3. The optimal points based on k-means.

\begin{tabular}{lll}
\hline & Longitude & Latitude \\
\hline 1 & 141.1327203 & 38.92887788 \\
2 & 141.1294003 & 39.74404741 \\
3 & 141.9601642 & 39.63154057 \\
4 & 141.8268725 & 39.25960077 \\
5 & 141.118439 & 39.29723965 \\
6 & 141.7661193 & 40.18740226 \\
7 & 141.718568 & 39.06258736 \\
8 & 141.138316 & 39.13352163 \\
9 & 141.1762277 & 39.69997165 \\
10 & 141.1588267 & 39.72299967 \\
11 & 141.1130611 & 39.3917413 \\
12 & 141.1486101 & 39.60799769 \\
13 & 141.1283003 & 39.69199232 \\
14 & 141.102124 & 39.73361104 \\
15 & 141.8773477 & 39.27300391 \\
16 & 141.1389663 & 39.77932715 \\
17 & 141.1589044 & 39.66004774 \\
18 & 141.9361671 & 39.64471253 \\
19 & 141.1649142 & 39.75616392 \\
\hline
\end{tabular}

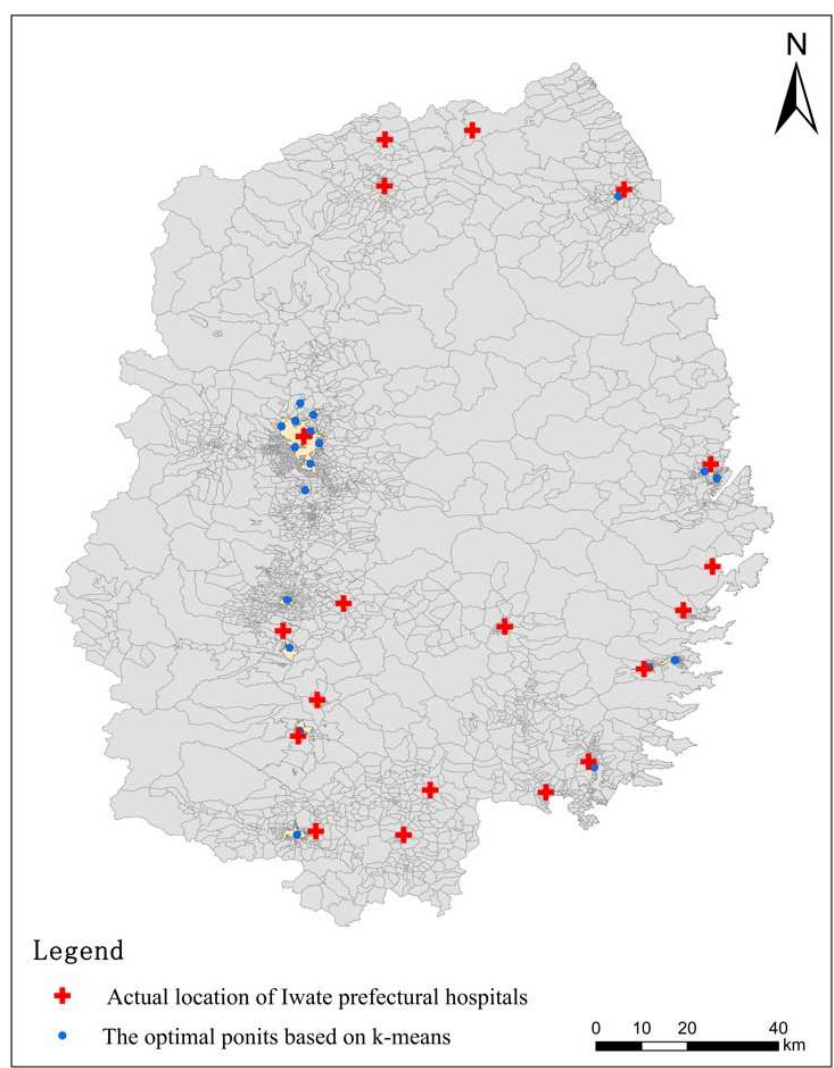

Figure 2. The optimal points based on k-means and actual locations of prefectural hospitals (The red dot is the actual location of the county hospital).

\subsection{Clustering Results of the Hospital Operating Index}

Using the cross-validation technique, the Calinski-Harabasz values were obtained for various parameters and the number of clusters is shown in Table 3. It is observed that the highest Calinski-Harabasz score is obtained when the number of clusters is 4 and the parameter is chosen 
as 10 . Table 4 represents the clustering results.

Table 4. The Calinski-Harabasz score of the cross-validation method.

\begin{tabular}{lll}
\hline parameter & clusters & Calinski-Harabasz score \\
\hline 0.01 & 2 & 25.9688 \\
0.01 & 3 & 20.8532 \\
0.01 & 4 & 15.9916 \\
0.1 & 2 & 28.8185 \\
0.1 & 3 & 20.8532 \\
0.1 & 4 & 15.9916 \\
1 & 2 & 28.8185 \\
1 & 3 & 56.7227 \\
1 & 4 & 45.4104 \\
10 & 2 & 25.9688 \\
10 & 3 & 34.5337 \\
10 & 4 & 71.8910 \\
15 & 2 & 24.8528 \\
15 & 3 & 34.5337 \\
15 & 4 & 71.8910 \\
\hline
\end{tabular}

Table 5. The clustering results of 19 prefectural hospitals.

\begin{tabular}{ll}
\hline 1 & Central \\
2 & Isawa, Iwai, Chubu \\
3 & Kamaishi, Ninohe, Kuji, Ofunato, Miyako \\
4 & Otsuchi, Takata, Towa, Ichinohe, Esashi \\
\hline
\end{tabular}

Based on the results of the above two clustering methods the following results can be pointed out:

(1) The results of cluster analysis of hospital operation indicators show that the 19 prefectural hospitals can be classified into four categories based on the number of beds, inpatients, nurses, and doctors.

(2) The first three groups are all in Iwate Prefectural Disaster Base Hospital.

(3) The first three groups are close to the clustering center of the k-means method, except for the prefectural Ninohe hospital. In other words, hospitals close to the population clustering centers acted relatively well in all aspects.

(4) Generally, hospital resources in Iwate Prefecture are unevenly distributed. Moreover, prefectural hospital resources are scarce although the population is concentrated in the Kamaishi secondary medical area. Considering prefectural hospital resources not private hospitals, it can be stated that prefectural medical resources are currently relatively scarce and can hardly deal with the relatively concentrated population.

\section{Discussion}

A medical evaluation method is proposed in this paper bringing novel ideas to the conception of medical care in Japanese regions. The method applies the k-means algorithm and spectral clustering algorithm. The model can be adjusted by altering the input variables' weights by the actual situation to monitor the allocation of medical resources in real-time. The results of the above analysis can be explained as follows.

There are 11 disaster critical hospitals in Iwate Prefecture, classified into primary disaster medical base hospitals and regional disaster medical base hospitals. Disaster base hospitals are hospitals receiving the sick and wounded. They dispatch medical rescue teams at the request of the governor of Iwate Prefecture in the event of a natural disaster when it is challenging to present proper medical care to victims under the standard medical system. The only two primary disaster medical base hospitals in Iwate Prefecture are located in the Morioka secondary medical area, namely Morioka Red Cross Hospital and Iwate Medical University Hospital. These two hospitals were not included in this study since the analysis was conducted on the prefectural hospitals. It was found that the remaining nine regional disaster stronghold hospitals are geographically close to the "best point" of the k-means method and belong to the top 3 groups in terms of clustering results of hospital indicators.

There is only one prefectural hospital in the Morioka medical area which is the best in all indicators. Moreover, the concentration of medical resources in the Morioka medical area is significantly higher than the population ratio. Because it is the prefectural office location, medical resources seem to be too concentrated. Moreover, the Morioka medical area should be regrouped to consider a more equitable distribution.

Although Ninohe Prefectural Hospital is not located in a population center, the hospital indicators acted relatively well. The reason is that, although cross-prefecture access has become possible in recent years as a result of advanced transportation, the geographical situation reveals that public hospitals predominate in the Ninohe medical area There are no private hospitals. Hence, it is challenging to "supplement" private hospitals facing the Ninohe medical area.

Furthermore, among the 10 hospitals ranked at the bottom of the management index, Daito Hospital, Otsychi Hospital, Takada Hospital, and Yamada Hospital were damaged in the Great East Japan Earthquake. As of October 2018, all of the prefectural hospitals affected by the disaster have been rebuilt. Doctors' support policies in the affected areas should be actively considered along with the reconstruction of the affected hospitals and the restoration of the number of hospital beds.

On September 26, 2019, the Japanese Ministry of Health, Labour, and Welfare summarized the results of an analysis revealing that 424 hospitals across Japan require special discussion on restructuring and mergers. It published a list of these hospitals including 10 national and public hospitals in Iwate Prefecture1. Four hospitals are prefectural including Prefectural Esashi Hospital in the Tanko medical area, Prefectural Towa Hospital in the Central medical area of Iwate Prefecture, Prefectural Ichinohe Hospital in the Ninohe medical area, and Prefectural Karumai Hospital. The clustering results in this study showed that the prefectural hospitals mentioned by the Ministry of Health, Labour, and Welfare as needing "restructuring and merging" were all those ranked at the bottom of the hospital management index. Hence,

\footnotetext{
1 The Nikkei Shimbun https://www.nikkei.com Accessed 8 Apr 2021.
} 
it can be stated that the method proposed in this study can present evidence for regional medical concept work.

This paper has the following points for improvement. First, only Iwate Prefectural Hospital was selected for analysis. To clarify the distribution of medical resources in the area, it is essential to include private hospitals in the analysis. There is also room for enhancement in the analysis method. In this paper, we focused only on the continuous model. However, the issue of the "robustness" of the analysis method must be considered since different facility allocation models have various decision criteria for the "optimal point". Furthermore, this paper used the k-means algorithm, which needs to be investigated with other algorithms in the future. At the time of writing this paper, a global pandemic of a novel coronavirus infection occurred. During our planned research, we attempted to conduct fieldwork. However, we had to stop it owing to the epidemic. Since the discussion was based on data published by hospitals, the analysis was inadequate. It is believed that this deficiency should be addressed as a topic for future research.

\section{Policy Recommendations}

In this paper, we propose the following policy recommendations:

(1) Building the healthcare service systems across secondary medical areas.

The clustering results of the hospital operating index show that prefectural medical resources in Iwate Prefecture are unevenly distributed, with more in the west and less in the east. The 2012 Iwate Prefecture patient's behavior survey suggests that the out-of-area patient's action rate was highest in the Ninohe medical area $(15.5 \%)$, followed by the Miyako medical area $(14.6 \%)$. In comparison, the 2017 out-of-area action rate was $18.1 \%$ in the Ninohe medical area and $17.6 \%$ in the Miyako medical area. It suggests that the number of visits across secondary medical areas is increasing and, therefore, the medical service systems across secondary medical areas should be constructed to improve the uneven distribution of medical resources.

(2) Administrative intervention to ensure the number of doctors in rural areas

The author believes that the main reason for the widening gap in medical resources in Iwate Prefecture is the uneven distribution of physicians. For example, Ofunato Hospital, located in the eastern part of the prefecture, has 489 beds and ranks second among 19 prefectural hospitals, but is in the third group of the hospital indicator cluster. It is owing to the low number of physicians, only 58.8 (the number of part-time physicians converted into the number of full-time physicians). There is a relationship between the number of physicians working in rural areas and the government. In order to ensure medical services in rural areas in the future, it is necessary to provide policy support for physicians working in the eastern part of Iwate Prefecture.

(3) When considering "public hospital restructuring," medical policies should be tailored to local conditions (e.g., the proportion of local public hospitals).

According to the clustering results, Ninohe prefectural hospital in the Ninohe medical area is relatively good in all indicators, though not located in the center of the cluster in the population concentration area. The reason is that the Ninohe medical area is dominated by public hospitals and supplemented by private hospitals. This is in contrast to the "private hospital provide the majority of medical care" characteristic of Japan. Therefore, the government should formulate a medical policy that is appropriate to the characteristics of the Ninohe medical area. For example, the opening of private hospitals is encouraged in the Ninohe Medical area.

\section{Conclusion}

In this paper, we applied the k-means algorithm and spectral clustering algorithm for clustering the data points of population concentration areas and prefectural hospital indicators in Iwate Prefecture, Japan. We verified the effectiveness of the combination of the two algorithms for evaluating regional medical resources using data from the Ministry of Land, Infrastructure, Transport and Tourism and the Hospital Information Bureau of Japan. This information will be effective in developing the national healthcare policies.

\section{References}

[1] Nomura, Hideki, and Takeo Nakayama. "The Japanese healthcare system." (2005): 648-649.

[2] Fushimi, K., and S. Matsuda. "Health resource reallocation by casemix data in Japan." BMC Health Services Research. Vol. 9. No. 1. BioMed Central, 2009.

[3] OECD. Hospital beds. https://data.oecd.org/healtheqt/hospital-beds.htm. Accessed 8 Apr 2021.

[4] Huang, Yihuai, et al. "Medical service demand forecasting using a hybrid model based on ARIMA and self-adaptive filtering method." BMC Medical Informatics and Decision Making 20.1 (2020): 1-14.

[5] Rechel, Berndl, et al. How can health systems respond to population ageing. No. EUR/08/5085883. Copenhagen: WHO Regional Office for Europe, 2009.

[6] Olanrewaju, Olawale, et al. "Physical activity in community-dwelling older people: a systematic review of reviews of interventions and context." PloS one 11.12 (2016): $\mathrm{e} 0168614$.

[7] Kim, Dong-Su, et al. "Telemedicine Center of Korean Medicine for treating patients with COVID-19: a retrospective analysis." Integrative medicine research 9.3 (2020): 100492.

[8] Moozhipurath, Rahul Kalippurayil, Lennart Kraft, and Bernd Skiera. "Evidence of protective role of Ultraviolet-B (UVB) radiation in reducing COVID-19 deaths." Scientific Reports 10.1 (2020): 1-10. 
[9] Mestre, Ana Maria, Mónica Duarte Oliveira, and Ana Paula Barbosa-Póvoa. "Location-allocation approaches for hospital network planning under uncertainty." European Journal of Operational Research 240.3 (2015): 791-806.

[10] Nagasaki Prefectural Medical Policy Division. https://www.pref.nagasaki.jp/section/iryo/ Accessed $8 \mathrm{Apr}$ 2021.

[11] Liu, Shirui, Yaochen Qin, and Yanan Xu. "Inequality and influencing factors of spatial accessibility of medical facilities in rural areas of China: a case study of Henan Province." International journal of environmental research and public health 16.10 (2019): 1833.

[12] Alsheikh, Mohammad Abu, et al. "Machine learning in wireless sensor networks: Algorithms, strategies, and applications." IEEE Communications Surveys \& Tutorials 16.4 (2014): 1996-2018.

[13] Hassani, Sahar, et al. "30-Day survival probabilities as a quality indicator for Norwegian hospitals: data management and analysis." PloS one 10.9 (2015): e0136547.

[14] Ng, Andrew, Michael Jordan, and Yair Weiss. "On spectral clustering: Analysis and an algorithm." Advances in neural information processing systems 14 (2001): 849-856.

[15] Nohara, Masaru. "Impact of the Great East Japan Earthquake and tsunami on health, medical care and public health systems in Iwate Prefecture, Japan, 2011." Western Pacific Surveillance \& Response Journal 2.4 (2011).

[16] Oliveira, Mónica Duarte, and Gwyn Bevan. "Modelling the redistribution of hospital supply to achieve equity taking account of patient's behaviour." Health care management science 9.1 (2006): 19-30.

[17] Rahman, Shams-ur, and David K. Smith. "Use of location-allocation models in health service development planning in developing nations." European Journal of Operational Research 123.3 (2000): 437-452.

[18] Matsumoto, Masatoshi, et al. "The impact of rural hospital closures on equity of commuting time for haemodialysis patients: simulation analysis using the capacity-distance model." International journal of health geographics 11.1 (2012): 1-10.

[19] Boonmee, Chawis, Mikiharu Arimura, and Takumi Asada. "Facility location optimization model for emergency humanitarian logistics." International Journal of Disaster Risk Reduction 24 (2017): 485-498.

[20] O'Kelly, Morton E. "The location of interacting hub facilities." Transportation Science 20.2 (1986): 92-106.

[21] O'Kelly, Morton E. "A clustering approach to the planar hub location problem." Annals of Operations Research 40.1 (1992): 339-353.

[22] MacQueen, James. "Some methods for classification and analysis of multivariate observations." Proceedings of the fifth Berkeley symposium on mathematical statistics and probability. Vol. 1. No. 14. 1967.

[23] McBratney, A. B., and J. J. De Gruijter. "A continuum approach to soil classification by modified fuzzy $\mathrm{k}$ - means with extragrades." Journal of Soil Science 43.1 (1992): 159-175.

[24] Wilpon, J., and L. Rabiner. "A modified K-means clustering algorithm for use in isolated word recognition." IEEE Transactions on Acoustics, Speech, and Signal Processing 33.3 (1985): 587-594.

[25] Duda, Richard O., and Peter E. Hart. Pattern classification and scene analysis. Vol. 3. New York: Wiley, 1973.

[26] Caliński, Tadeusz, and Jerzy Harabasz. "A dendrite method for cluster analysis." Communications in Statistics-theory and Methods 3.1 (1974): 1-27. 